\title{
THE EXISTENCE OF A TOTAL ORDER ON A GROUTP
}

\author{
E. J. TULLY, JR.
}

We call a subset $S$ of an ordered set right isolated if whenever $x \in S$ and $x \leqq y$ it follows that $y \in S$. Theorem 1 gives a necessary and sufficient condition for an orderable group $G$ to be orderable in such a way that a given subset of $G$ is right isolated. This result is used to obtain, in Theorem 2, a new necessary and sufficient condition for a group to be orderable. The condition is stated in two forms, and is somewhat similar to a condition obtained by Ohnishi [2].

Several authors, e.g., Conrad [1], have introduced the concept of right-ordered group (a group with a total order which is assumed to satisfy the right-handed monotone condition: $a c \leqq b c$ whenever $a \leqq b)$. In Theorems $1^{\prime}$ and $2^{\prime}$ of the present note we state the "righthanded" versions of Theorems 1 and 2; the proofs (here omitted) are similar.

Professor Paul Conrad has made valuable suggestions concerning the subject matter of this note. In particular, he suggested Theorems $1^{\prime}$ and $2^{\prime}$. The writing of the final version was supported by the National Science Foundation.

Theorem 1. Let $G$ be a group which can be ordered. Let $S$ be any subset of $G$. There exists an order on $G$ for which $S$ is right isolated if and only if for all $g, h \in G$ either $g S h \subseteq S$ or $S \subseteq g S h$, where $g S h$ $=\{g x h: x \in S\}$.

Proof. First, suppose our condition is not satisfied. Then there exist $x \in S$ with $g x h \notin S$, and $y \notin S$ with $g y h \in S$. Now if $\leqq$ is any order on $G$, either $x \leqq y$ (and hence $S$ is not right isolated) or $y \leqq x$ (so that $g y h \leqq g x h$, and hence $S$ is not right isolated).

Conversely, suppose our condition is satisfied. Define $J=\{x \in G: a b \in S$ implies $a x b \in S$, for all $a, b \in G\}$. Let $P$ be the set of positive elements under any order on $G$. Let $P^{\prime}=\{x \in G$ : either $x \in J, x^{-1} \in J, x \in P$ or $\left.x \in J, x^{-1} \notin J\right\}$. It is easy to verify that $J$ is a normal subsemigroup. We now show that for all $x \in G$ either $x \in J$ or or $x^{-1} \in J$. Suppose $x \notin J$. Then $a b \in S, a x b \in S$, for some $a, b \in G$. Let $c d \in S$ be given. Then $a x c^{-1} S d^{-1} b \subseteq S$ does not hold, since $a x c^{-1} c d d^{-1} b$ $=a x b \in\left(a x c^{-1} S d^{-1} b\right) \backslash S$. Hence $S \subseteq a x c^{-1} S d^{-1} b$, so that $c x^{-1} a^{-1} S b^{-1} d$ $\subseteq S$. Hence $c x^{-1} d=c x^{-1} a^{-1} a b b^{-1} d \in S$. Thus, we have shown that $x^{-1} \in J$. Now suppose $x, y \in P^{\prime}$. Then either $x \in J, x^{-1} \in J, x \in P$, $y \in J, y^{-1} \in J, y \in P$ (in which case $x y \in J, y^{-1} x^{-1} \in J, x y \in P$, so that

Received by the editors February 1, 1961. 
$x y \in P^{\prime}$ ), or $x \in J, x^{-1} \in J, y \in J$ (in which case it can be seen that $x y \in J, y^{-1} x^{-1} \in J$, so that $x y \in P^{\prime}$ ), or $x \in J, y \in J, y^{-1} \in J$ (in which case $x y \in P^{\prime}$, by reasoning similar to that employed in the preceding case). Thus we have shown that $P^{\prime}$ is a semigroup. The fact that $P^{\prime}$ is normal follows from the corresponding fact for $J$. Clearly $e \notin P^{\prime}$, and it is easy to check that for all $x \neq e$ either $x \in P^{\prime}$ or $x^{-1} \in P^{\prime}$. Thus, we have shown that $P^{\prime}$ is the set of positive elements for an order $\leqq$ defined by: $x \leqq y$ if and only if $y x^{-1} \in P^{\prime}$. Clearly, $S$ is right isolated relative to this order (for if $x \in S$ and $y x^{-1} \in P^{\prime}$, we have $y x^{-1} \in J$, and hence $\left.y=e\left(y x^{-1}\right) x \in S\right)$.

THEOREM 2. Let $G$ be a group. The following are equivalent:

(1) $G$ can be ordered.

(2) For all $g \in G$ with $g \neq e$, there exists $S \subseteq G$ with $g \in S, e \in S$ and for all $g^{\prime}, h^{\prime} \in G$ either $g^{\prime} S h^{\prime} \subseteq S$ or $S \subseteq g^{\prime} S h^{\prime}$.

(3) There exists a family $\Sigma$ of subsets of $G$ such that $e=\bigcap_{S \in \Sigma} S$ and for all $g, h \in G$ and $S \in \Sigma$ either $g S h \subseteq S$ or $S \subseteq g S h$.

Proof. First suppose (1) is satisfied and let $\leqq$ be an order on $G$. Given $g \neq e$, let $S$ be either $\{x: g \leqq x\}$ or $\{x: x \leqq g\}$, according as $e<g$ or $g<e$. Then, by Theorem $1, S$ satisfies the conditions of (2).

Next, if (2) is satisfied, for each $g \neq e$ choose a set $S_{0}$ satisfying the conditions of (2). Let $\Sigma=\left\{G \backslash S_{x}: x \in G, x \neq e\right\}$. It is easy to see that $\Sigma$ satisfies (3).

Finally, suppose (3) is satisfied. For each $S \in \Sigma$ let $J(S)=\{x \in G$ : $a b \in S$ implies $a x b \in S$, for all $a, b \in G\}$, as in the proof of Theorem 1 . Then, as we have seen, $J(S)$ is a normal subsemigroup and for all $x \in G$ either $x \in J(S)$ or $x^{-1} \in J(S)$. Well order $\Sigma$. Let $P=\{x \in G$ : for some $S \in \Sigma, x \in J(S)$ and $x^{-1} \in J(S)$, while for all $T<S, x \in J(T)$ and $\left.x^{-1} \in J(T)\right\}$. It is easy to check that $P$ is a normal subsemigroup not containing $e$. We assert that for all $x \neq e$ either $x \in P$ or $x^{-1} \in P$. For otherwise we would have $x, x^{-1} \in J(S)$ for all $S \in \Sigma$. Hence, by definition of $J$ (since $e \in S$ ) we have $x=e x e \in S$, for all $S \in \Sigma$. Hence $x \in \cap_{s \in \Sigma} S$, so that by (3) $x=e$. Thus we have shown that $P$ is the set of positive elements for some order on $G$.

THEOREM $1^{\prime}$. Let $G$ be a group which can be right-ordered. Let $S$ be any subset of $G$. There exists a right-order on $G$ for which $S$ is right isolated if and only if for all $g \in G$ either $S g \subseteq S$ or $S \subseteq S g$, where $S g$ $=\{x g: x \in G\}$.

THEOREM $2^{\prime}$. Let $G$ be a group. The following are equivalent:

(4) $G$ can be right-ordered.

(5) For all $g \in G$ with $g \neq e$, there exists $S \subseteq G$ with $g \in S, e \notin S$ and 
for all $g^{\prime} \in G$ either $S g^{\prime} \subseteq S$ or $S \subseteq S g^{\prime}$.

(6) There exists a family $\Sigma$ of subsets of $G$ such that $e=\bigcap_{s \in \Sigma} S$ and for all $g \in G$ and $S \in \Sigma$ either $S g \subseteq S$ or $S \subseteq S g$.

\section{REFERENCES}

1. P. Conrad, Right-ordered groups, Michigan Math. J. 6 (1959), 267-275.

2. M. Ohnishi, Linear-order on a group, Osaka Math. J. 4 (1952), 17-18.

California Institute of Technology

\section{NONEXTENDIBILITY CONDITIONS ON MUTUALLY ORTHOGONAL LATIN SQUARES}

\section{E. T. PARKER}

In this note are proved two theorems which impose restrictions on the structure of sets of mutually orthogonal latin squares. The corollary yields a version stronger in a certain direction than a known theorem on possible orders of subplanes of finite projective planes. This work developed from study of the first pairs of orthogonal latin squares of order 10, all of which had a pair of orthogonal latin subsquares of order $3([1 ; 2$, pp. 198-200]; another unpublished construction was found by Bose and Shrikhande). There exist pairs of orthogonal latin squares of order 10 having no pair of orthogonal latin subsquares of order 3 ; examples were generated with UNIVAC M-460 Computer, programmed by the author [3]. The arguments in this note depend on inequalities. A theorem of Mann $[4 ; 5]$ on orthogonal latin squares of orders $4 t+2$ was proved using inequalities and parities. The author feels that some combination of his own and Mann's methods might lead to better theorems.

THEOREM 1. If a set of $t$ mutually orthogonal latin squares (m.o.l.s.) of order $n$ has a set of $t$ mutually orthogonal latin subsquares (m.o.l. subs.) of order $r$, with $r<n$, then $n \geqq(t+1) r$.

Proof. Partition the rows and columns of the m.o.l.s. into sets of $n-r$ and $r$, with the m.o.l. subs. contained in the sets of $r$ rows and $r$ columns. Designate the $(n-r) \times(n-r)$ block by $A$, the $r \times r$ block by $D$, and the two rectangular blocks by $B$ and $C$. Let $x_{1}, \cdots, x_{r}$

Presented to the Society, January 28,1960 ; received by the editors January 17 , 1961. 